\title{
Colorectal carcinoma in patients with ulcerative colitis and recent colonoscopy
}

\author{
S M Catnach, K R P Rutter, R L Bown
}

\begin{abstract}
Longstanding ulcerative colitis, particularly pancolitis, is associated with an increased risk of colorectal neoplasia. For this reason surveillance colonoscopy at regular intervals has been recommended to identify early cancers or high grade dysplasia. Three cases are described of patients with ulcerative colitis of greater than 10 years duration who presented with colorectal carcinoma within three years of colonoscopy.
\end{abstract}

(Gut 1993; 34: 1148-1149)

\section{Case 1}

A 28 year old woman with a 23 year history of ulcerative colitis. She was receiving oral sulphasalazine and intermittent courses of oral prednisolone and depot adrenocorticotrophic hormone injections. In 1980 a barium enema showed total colitis and benign strictures in the transverse and ascending colon. Colonoscopy in 1981 confirmed these findings with biopsy specimens of the mucosa showing active ulceration throughout the colon but no evidence of dysplasia. A repeat colonoscopy in 1983 was unchanged. In 1984, however, she presented with abdominal pain. Rigid sigmoidoscopy showed a benign looking stricture in the rectum, which was not examined by biopsy. Her symptoms did not settle and seven months later she had a total colectomy. Histological examination of the specimen showed the rectal stricture to be a Dukes's stage A carcinoma with no dysplasia in the remaining colon.

\section{Case 2}

A 74 year old woman with a 30 year history of ulcerative colitis. In 1974 a barium enema showed total colitis with a shortened colon. In 1990 she returned to the outpatient clinic complaining of worsening symptoms. A colonoscopy performed in May 1991 showed total colitis and inflammatory polyps. Histological examination of the mucosal biopsy specimens confirmed active colitis with mild dysplasia. Persistent symptoms led to colectomy in July 1992. Pathological examination showed two carcinomas; an ulcerated $1.5 \mathrm{~cm}$ polyp in the ascending colon with invasive carcinoma and a plaque like carcinoma in the transverse colon.

\section{Case 3}

A 72 year old man with distal colitis for 29 years. After prolonged treatment with oral sulphasalazine he was reassessed in the outpatient clinic in 1987. A total colonoscopy at that time showed quiescent left sided colitis with no dysplasia on biopsy examination of the mucosa. In 1990 he complained of alternating constipation and diarrhoea and tenesmus. Rigid sigmoidoscopy was performed and a nodular area in the rectum examined by biopsy. This proved to be a moderately differentiated adenocarcinoma and the patient had panproctocolectomy and ileostomy.

\section{Comment}

There is undoubtedly an increased risk of carcinoma in patients with a long history of extensive colitis, and it is probably about $0.5-1 \%$ per person per year in those with pancolitis for 20 years or more. ' In an attempt to reduce this risk, regular surveillance colonoscopy has been recommended for those designated to be at particularly high risk (extensive colitis for greater than 10 years). The aim of screening colonoscopy in these patients is to identify cancers at an early stage, when prognosis is more favourable and to detect high grade dysplasia, which is assumed to progress to cancer. Evidence that screening is effective in increasing survival is lacking, however, and even patients enrolled in screening programmes still develop cancers - either because cancer or dysplasia was missed at an earlier examination or because cancer developed in the interval between examinations.

The frequency of surveillance colonoscopy in longstanding ulcerative colitis is not established. Annual or biennial examinations have been recommended, although even with six monthly examinations early cancers were still being discovered. ${ }^{2}$ It is interesting that the patients we have reported on all had no or mild dysplasia only, as carcinoma in ulcerative colitis is usually thought to be associated with the presence of high grade dysplasia. A recent report would suggest that low grade or mild dysplasia is also associated with the development of carcinomas and our three cases would support this. ${ }^{3}$ Two of the four carcinomas were plaque like or carpet lesions - a morphological type associated with inflammatory bowel disease. These are difficult to identify at endoscopy as they may seem to be merely a rather roughened area of mucosa. One of the four was a fairly small polyp, again easily missed at colonoscopy.

These three cases highlight several important points. Firstly, that colonoscopy is fallible in the detection of tumours; the lesions in cases 1 and 2 must have been present at the time of colonoscopy despite being examined by an experienced endoscopist. Secondly, mild or low grade dysplasia on random biopsy of the colon is misleading; our cases show that these patients are still at risk of malignancy. This discrepancy may partly be accounted for by difficulties in the pathological grading of dysplasia, which may 
show wide interobserver variation. ${ }^{+}$As our first case shows, however, cancers can occur even when histological examination of the whole colon has failed to detect dysplasia of any degree.

Collins, et al have elegantly reviewed the whole problem of surveillance colonoscopy in ulcerative colitis and put surveillance in perspective, both in terms of cost, and benefit to the patient. ${ }^{5}$ They estimate that (in the United States in 1987) about $\$ 200000$ would be spent for each cancer found or prevented and go on to conclude that 'the premises on which the argument for surveillance is based are not well established' and that 'there is no compelling evidence that surveillance is beneficial'.

If screening of patients with longstanding ulcerative colitis is offered to patients, these points must be appreciated and the patient advised that surveillance colonoscopy does not remove the threat of cancer.

We wish to thank the Joint Research Board of St Bartholomew's Hospital for financial support to Dr Catnach.

1 Ransohoff DF. Colon cancer in ulcerative colitis. Gastroenterology 1988; 94: 1089-91.

2 Lennard-Jones JE. Compliance, cost, and common sense limit cancer control in colitis. Gut 1986; 27 : 1403-7.

3 Woolrich AJ, DaSilva MD, Korelitz BI. Surveillance in the routine management of ulcerative colitis. The predictive value of low-grade dysplasia. Gastroenterology 1992; 103: 4318.

4 Butt JH, Price A, Williams CB. Dysplasia and cancer in ulcerative colitis. In: Allan RN, Norman R, eds. Inflammatory
bowel diseases. Edinburgh: Churchill Livingstone, 1983; 14053.

5 colon

Colon cancer, dysplasia, and surveillance in patients with ulcerative colitis. A critical review. Collins RH, Feldman M, Fordtran JS. N Engl f Med 1987; 316: 1654-8. 